\title{
ANÁLISE DA CINÉTICA DE REMOÇÃO DE LACTATO EM ATLETAS DE CANOAGEM SLALOM
}

\section{MS. CARLA CRISTIANE DA SILVA}

Docente do Departamento de Educação Física da Universidade Estadual do Norte do Paraná - UENP e Grupo de Estudos das Adaptações Fisiológicas ao Treinamento (GEAFIT), Centro de Educação Física e Esporte da Universidade Estadual de Londrina

(Londrina - Paraná - Brasil)

E-mail: ccsilva@uenp.edu.br

\section{ESP. MILENE WOLFF}

Técnica de Canoagem Slalom e especialista em Personal Trainer pela

Universidade Estadual do Norte do Paraná (UENP) (Piraju - São Paulo - Brasil)

E-mail: milenewolff@hotmail.com

\section{MS. CLODOALDO JOSÉ DECHECHI}

Laboratório de Bioquímica do Exercício (LABEX), Instituto de Biologia - IB - Universidade Estadual de Campinas - UNICAMP (Campinas - São Paulo - Brasil)

E-mail: cdechechi@yahoo.com.br

\section{MS. ALEXANDRE GOMES DE ALMEIDA}

Mestre em Ciências da Motricidade pela Universidade Estadual Paulista (UNESP-Rio Claro)

Docente da Faculdade de Educação Física no Centro de Ciências Humanas e Sociais Aplicadas da Pontifícia Universidade Católica de Campinas (PUC-Campinas)

(Campinas - São Paulo - Brasil)

E-mail: alexandrealmeida74@hotmail.com

\section{DR. FÁBIO YUZO NAKAMURA}

Grupo de Estudos das Adaptações Fisiológicas ao Treinamento (GEAFIT) Centro de Educação Física e Esporte da Universidade Estadual de Londrina (Londrina - Paraná - Brasil)

E-mail: fabioy_nakamura@yahoo.com.br

\section{RESUMO}

O objetivo do presente estudo foi verificar a cinética de produção e remoção de lactato em atletas de canoagem slalom da categoria Kl, durante competição oficial. Participaram do estudo oito atletas do sexo masculino (22,6 \pm 4,3 anos). Foram realizadas coletas de $25 \mu \mathrm{L}$ de sangue capilar para análise do lactato. A cinética de remoção do lactato foi realizada antes do aquecimento (Pré), logo após a saída dos competidores do rio (Pós 0'), 5 (Pós 5') e 20 (Pós 20') minutos. Os resultados demonstraram um aumento significativo nas concentrações de lactato (9,8 mmol/l, 9,4 mmol/l e 6,6 mmol/l) nos tempos 0', 5' e 20' minutos 
pós respectivamente, com valores de $P<0,01$. Os achados indicam que após 20 minutos, os valores de lactato se reduziram de forma importante $(P<0,05)$ em relação aos valores pré exercício (2,0 mmol/l), sugerindo que os atletas indicariam boas condições metabólicas para a segunda descida da prova.

PALAVRAS-CHAVE: Lactato sanguíneo; exercícios de alta intensidade; competição; atletas.

\section{INTRODUÇÃO}

A canoagem slalom é uma modalidade disputada em rios com corredeiras, sendo que o canoísta deve passar por barreiras, a favor ou contra a correnteza, não podendo tocá-las. Cada atleta realiza o percurso em duas descidas, com intervalo de 90 a 120 minutos (ZAMPARO et al., 2006; MICHAEL et al. , 2009; NIBALLI et al., 20 I I). Para obter sucesso, os atletas devem planejar, com técnica apurada e potência muscular, a rota mais apropriada para atravessar as balizas (KEARNEY; MCKINSEY, 2000; MELLO et al., 2009; GARCÍA-PALLARÉS et al., 20 I 0), contemplando uma combinação de breves acelerações e remadas no máximo de velocidade (MICHAEL et al. , 201 2), evitando receber penalizações (HUNTER, 2009; NIBALLI et al. , 20 I I).

Estudos indicam que atletas desta modalidade dispõem de valores elevados de capacidade anaeróbia e força muscular de membros superiores (BISHOP, 2000a; NAKAMURA et al., 2006; AKCA; MUNIROUGLU, 2008; MICHAEL et al. , 2009; GARCÍA-PALLARÉS; IZQUIERDO, 20II). Os atletas, além de dispor, na maior parte do tempo, de treinamento para aperfeiçoamento da técnica, também enfatizam adaptações de ordem cardiorrespiratória e neuromusculares, buscando o desenvolvimento da capacidade oxidativa, além de potência muscular de membros superiores (KEARNEY; MCKINSEY, 2000; BISHOP, 2000a; ZAMPARO et al. , 2006; GARCÍA-PALLARÉS et al. , 2009; GARCÍA-PALLARÉS; IZQUIERDO, 20I I).

Especificamente na modalidade de canoagem slalom, uma prova competitiva de alta intensidade e curta duração, exige-se extrema demanda energética; logo, a ressíntese de ATP ocorre em grande magnitude à custa do metabolismo anaeróbio (BISHOP; CLAUDIUS, 2005; KEMP et al., 2006; MACIEJEWSKI et al., 20I2). Durante este tipo de atividade a produção de lactato e $\mathrm{H}^{+}$é muito rápida, porém, a liberação do músculo é lenta (GLADDEN, 2000; JUEL et al. , 2004; KEMP et al., 2006), resultando no aumento intracelular e no sangue de lactato e de $\mathrm{H}^{+}$. Para ser utilizado, o lactato produzido na fibra muscular deve ser transportado para a corrente sanguínea. Para isto, uma proteína presente na membrana da célula muscular, conhecida como transportador de monocarboxilato (MCT), transporta o lactato de dentro da célula para o sangue (JUEL et al. , 2004; BONEN et al., I998; BONEN, 2000; GLADDEN, 2007; BISHOP et al., 2008). O transporte do lactato 
para a corrente sanguínea através dos MCTs somente é realizado em co-transporte com H $\mathrm{H}^{+}$, constituindo-se, dessa forma, como um dos processos que mais contribui para a manutenção do pH intracelular (BISHOP et al., 200 I; JUEL et al., 2004; THOMAS et al., 2005; GLADDEN, 2007; BISHOP et al., 2008); BOGDANIS, 20 I2).

Neste contexto, estudos prévios reportados na literatura objetivaram analisar a cinética de remoção de lactato frente a esforços de alta intensidade (BRET et al., 2003; MESSONNIER et al., 2006; BISHOP et al. 2008; MENZIES et al., 20 I0). A produção de lactato indica a magnitude da capacidade de produção de ATP via metabolismo anaeróbico lático, e sua remoção para o sangue uma capacidade de regulação de $\mathrm{pH}$ intramuscular. Já a remoção do sangue é um indicativo da capacidade de re-utilização deste como substrato energético em tecidos como o fígado, coração, e fibras do tipo I (CABRERA et al., 1999; CERRETELLI; SAMAJA, 2003; JUEL et al., 2004; CAIRNS, 2006; MACIEJEWSKI et al., 20 I2).

Sendo assim, a quantificação/avaliação das capacidades de produção e remoção de lactato é um dos pontos determinantes para o desempenho em modalidades esportivas intermitentes, como a canoagem slalom. Contudo, raros são os estudos na literatura sobre a modalidade (NIBALLI et al., 20 I I), particularmente na quantificação do resultado metabólico dos esforços durante evento competitivo. Deste modo, o objetivo do estudo foi descrever a cinética de produção e remoção de lactato em atletas de canoagem slalom da categoria KI , após a primeira descida do percurso de uma competição oficial da modalidade.

\section{MATERIAIS E MÉTODOS}

\section{AMOSTRA}

O presente estudo foi realizado na $4^{\text {a }}$ Etapa da Copa Brasil de Canoagem Slalom, no município de Piraju-SP. Esta etapa foi escolhida por sua importância na classificação dos atletas no cenário nacional e, principalmente, pelas características do Rio Paranapanema, onde a prova foi realizada, considerada a mais complexa pista natural de slalom no país.

Nesta etapa foram inscritos 8 atletas adultos do sexo masculino na modalidade KI, caracterizados por remar, sentados individualmente, no caiaque. Todos foram convidados a participar, e consentiram após explanação detalhada anterior a qualquer procedimento de coleta.

Os atletas foram devidamente informados sobre os procedimentos adotados na pesquisa, tendo assinado um termo de consentimento livre e esclarecido. Foi firmado o compromisso de entrega individual dos resultados para cada atleta, assim 
como um relatório geral para Confederação Brasileira de Canoagem (CBCa) que, conjuntamente com a Organização do Evento, autorizaram a realização do estudo. A pesquisa foi aprovada pela Comissão de Ética em Pesquisa da Universidade do Norte do Paraná (080/20 I0).

\section{CARACTERIZAÇÃO DA PISTA DE COMPETIÇÃO}

A pista de competição foi estruturada com um percurso de 250 metros, com a colocação de dezessete portas a favor da corrente e nove portas conhecidas como remontas, contra a correnteza. Informações no dia da competição indicaram que o Rio Paranapanema estava com um fluxo de $190 \mathrm{~m}^{3} / \mathrm{s}$, que é muito superior à média internacional de $10 \mathrm{~m}^{3} / \mathrm{s}$. No dia da competição as condições ambientais indicaram uma temperatura de 26 a $28^{\circ} \mathrm{C}$, dia ensolarado, intensidade do vento $17 \mathrm{~km} / \mathrm{h}$, direção do vento Norte-Nordeste e umidade de $50 \%$.

\section{AVALIAÇÃO ANTROPOMÉTRICA}

Foi realizada avaliação antropométrica dos atletas às 08:00 h da manhã no dia da competição, duas horas antes do aquecimento para a primeira descida do percurso. A avaliação antropométrica foi realizada com objetivo de caracterizar, de forma geral, o grupo de atletas da modalidade de slalom. Foram tomadas as dobras cutâneas nas regiões tricipital, subescapular, peitoral, axilar média, suprailíaca, abdominal e coxa, de acordo com os procedimentos descritos por HARRISSON et al. (1988). Todas as medidas foram realizadas por um único avaliador experiente, de forma rotacional, e replicadas três vezes, sendo registradas por um anotador. A partir dos valores das espessuras de dobras cutâneas, foi calculada a densidade corporal, empregando-se equações preditivas propostas por Jackson e Pollock ( 1978), e posteriormente realizado o cálculo da gordura corporal relativa estimada pelas equações propostas por SIRI ( 1961 ).

\section{PROTOCOLO DE INVESTIGAÇÃO}

Foram realizadas coletas sanguíneas de repouso para análise do lactato sanguíneo exatamente I hora antes do início da competição. Foram coletados aproximadamente $25 \mu \mathrm{L}$ de sangue por capilar do lóbulo da orelha com o uso de lancetas descartáveis marca Feathe. Para as análises foi utilizado lactímetro portátil (Accusport ${ }^{\circledR}$ - Boeringer Mannheim), anteriormente descrito e validado (FELL et al., 1998; INTERDONATO; LUETKEMEIER, 1999). A cinética de remoção do lactato foi realizada em quatro momentos distintos: anteriormente ao aquecimento 
(medida de repouso- valores Pré), logo após a saída dos competidores do rio (Pós 0'), 5 minutos depois (Pós 5') e 20 minutos após a coleta Pós 0' (Pós 20').

\section{ESTATÍSTICA}

A normalidade dos dados foi averiguada através do teste de Shapiro-Wilk. Para análise de diferença entre as médias, foi utilizado o teste ANOVA. Assumiu-se nível referência significativa de $P<0,05$. Posteriormente avaliou-se o cálculo do tamanho do efeito de Cohen (ES) (COHEN, 1988) utilizado para interpretar as diferenças nas médias entre os momentos, com a escala adaptada por Hopkins (www.sportsci. org/resource/stats) (HOPKINS et al., 2009), sendo utilizada para a interpretação: < 0.2 Trivial; 0.2 - 0.6 Pequeno; 0.6 - 1.2: Moderado; > 1.2 Grande.

\section{RESULTADOS}

A Tabela I apresenta os valores médios \pm desvio padrão dos indicadores antropométricos dos sujeitos. Os atletas que participaram do estudo, além da experiência em competições nacionais e internacionais (6,8 \pm 2,7 anos de experiência profissional), relataram que a preparação para a prova foi de no mínimo seis meses, com um treinamento semanal total 9,0 \pm 3,7 horas. $\bigcirc$ tempo de execução do percurso na primeira descida dos oito atletas foi médio de 106,8 \pm 22 ,8 segundos.

Tabela I. Caracterização antropométrica do grupo de atletas de canoagem slalom do sexo masculino $(n=8)$

\begin{tabular}{lccc}
\hline & Média \pm DP & Mínimo & Máximo \\
\hline Idade (anos) & $22,6 \pm 4,3$ & 18,0 & 31,0 \\
Experiência profissional (anos) & $6,8 \pm 2,7$ & 5,0 & 12,0 \\
Treino semanal (horas) & $9,0 \pm 3,7$ & 5,0 & 15,0 \\
Massa Corporal (kg) & $70,6 \pm 9,2$ & 54,3 & 84,3 \\
Estatura $(\mathrm{m})$ & $1,77 \pm 0,05$ & 1,66 & 1,84 \\
IMC $\left(\mathrm{kg} / \mathrm{m}^{2}\right)$ & $22,4 \pm 2,2$ & 19,5 & 25,4 \\
Envergadura $(\mathrm{cm})$ & $1,82 \pm 0,04$ & 1,74 & 1,89 \\
Tórax $(\mathrm{cm})$ & $96,1 \pm 5,6$ & 88,2 & 106,0 \\
Abdômen $(\mathrm{cm})$ & $80,5 \pm 5,1$ & 71,3 & 85,9 \\
Quadril $(\mathrm{cm})$ & $92,5 \pm 6,1$ & 80,0 & 97,2 \\
MCM $(\mathrm{kg})$ & $63,8 \pm 7,6$ & 51,3 & 77,2 \\
Peso Gordura $(\mathrm{kg})$ & $6,87 \pm 2,5$ & 3,0 & 10,0 \\
Gordura relativa $(\%)$ & $9,61 \pm 2,9$ & 5,5 & 13,2 \\
\hline Nota Resutados & &
\end{tabular}

Nota. Resultados são expressos em média \pm SD 
A Figura I apresenta os valores médios \pm desvio padrão das concentrações de lactato nos momentos indicados. Observamos diferença significativa $(P<0,0$ I $)$ dos momentos 0 ' pós e 5' pós em relação ao momento pré. Foi observada também diferença significativa do momento 20' pós em relação ao momento pré. Entre o momento pós 20' e pós 5' aplicou-se estatística de Cohen para magnitude do efeito, e a interpretação das diferenças nas médias entre os momentos foi realizada com a escala adaptada por Hopkins. Os resultados indicaram que entre o momento pós 5' e pós 20' houve uma redução de 9,4 para 6,6 mmol, classificada como efeito moderado $(-0,63)$.

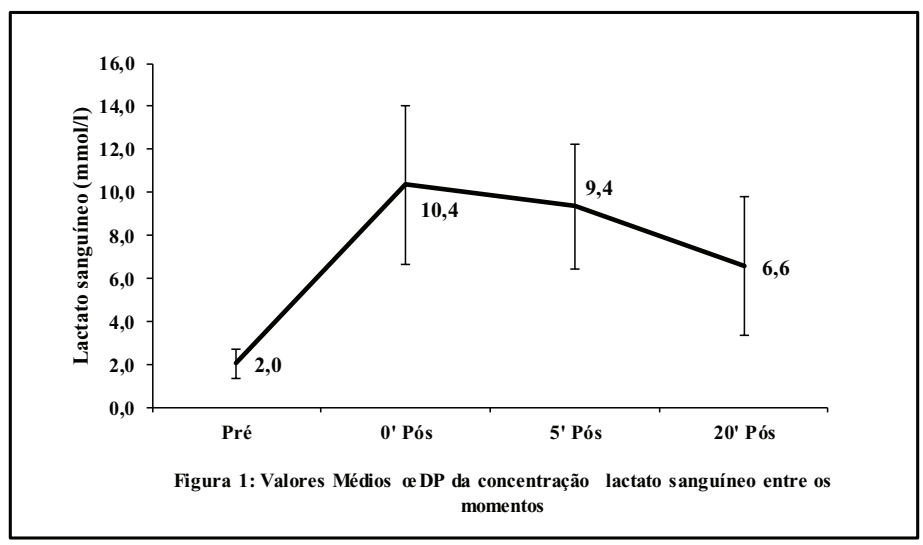

\section{DISCUSSÃO}

Embora a canoagem slalom seja uma modalidade olímpica desde 1972, raras são as investigações na modalidade com foco nos aspectos fisiológicos. No Brasil as pesquisas se direcionaram para canoagem velocidade (HENSEL et al., 2008; NAKAMURA et al., 2007; 2008; MOREIRA et al., 2009; LEMOS et al., 20l0) e, como são modalidades distintas, o presente estudo vem com a perspectiva de indicar os primeiros relatos da cinética de produção e remoção de lactato durante uma importante competição nacional. Priorizar o evento competitivo, no caso específico da canoagem slalom, é garantir uma análise ecológica, no sentido de refletir de fato o que ocorre com o atleta durante a situação competitiva. Por outro lado, uma grande parcela das investigações ocorre no ambiente laboratorial, utilizando ergômetros, onde é possível o maior controle de variáveis fisiológicas (BISHOP et al., 200 I; MELLO et al., 2009; MICHAEL et al., 20 I0; NIBALI et al., 20I I; SEALEY et al., 2010; GOMES et al., 2012), entretanto, com um certo prejuízo 
na transferência dos resultados para situações de campo. Neste contexto, descrito recentemente por Michael e colaboradores (20 I2), a melhor situação de avaliação ocorre no ambiente natural de competição, entretanto é complexo o controle de múltiplas variáveis intervenientes. Um modelo interessante para investigar o treinamento e suas repercussões frente aos resultados competitivos foi realizado por García-Pallarés e colaboradores (20 I 0a). Os autores estudaram, durante duas temporadas, dois modelos de periodização de treinamento, tradicional e bloco, em um grupo de canoístas de elite, todos finalistas do Campeonato Mundial, incluindo dois medalhistas olímpicos. Foram realizados teste incremental em kayak ergômetro com avaliação do $\mathrm{VO}_{2}$ pico, limiar ventilatório e concentração de lactato no I , 3ㅇ, 5, 7, 10 e $12 \circ$ de recuperação. Os resultados apontaram que a periodização em bloco foi mais efetiva do que a modelagem tradicional e, com relação aos valores de lactato na recuperação após teste incremental, não foram observadas diferenças entre os modelos de treinamento, com valores médios ligeiramente superiores ao presente estudo (10- 13 mmol//) no momento 0' pós.

Estudos com diferentes intervenções de treinamento são fundamentais no sentido de responderem questões da prática do treinamento físico, no entanto, o presente estudo teve como característica descrever as primeiras evidências referentes à canoagem slalom no Brasil, e, embora seja análise aguda de desempenho esportivo durante prova competitiva, os atletas incluídos fazem parte de um grupo seleto na canoagem slalom, todos com participações e bons resultados em campeonatos brasileiros, e 50\% deles com participações em etapas mundiais.

As características antropométricas e da composição corporal foram descritas na tabela I . É possível ressaltar que os atletas brasileiros apresentaram valores médios similares aos encontrados por Zamparo et al. (2006), com 8 atletas de KI para as variáveis de massa corporal, estatura, IMC, com exceção apenas para a gordura relativa, sendo $7,7 \%$ e $9,6 \%$, respectivamente. O perfil antropométrico de canoístas da seleção da Turquia foi investigado por Akca e Munirouglu (2008), e os resultados apontaram peso corporal e percentual de gordura superiores aos apresentados no presente estudo. De forma geral, investigações mais recentes com atletas de elite do sexo masculino, embora não específicas de canoagem slalom, indicaram atletas de faixa de idade similar, contudo, com valores superiores de massa corporal e estatura, de aproximadamente $85 \mathrm{~kg}$ e I,80cm, respectivamente (MELLO et al., 2009; MICHAEL et al. 20 I0; GARCIA-PALLARÉS et al., 20 I0; MICHAEL et al., 20।2).

Com relação à avaliação da cinética de lactato sanguíneo, obtêm-se indicadores quantitativos do curso temporal no qual o lactato se encontra acumulando no compartimento sanguíneo. Foi sugerido que a produção de lactato pela via glicolítica, por meio da ação da enzima lactato desidrogenase e, consequentemente, sua 
remoção, na realidade, contribuiria para manutenção do pH intramuscular (ROBERGS, 200 I; ROBERGS, GHIASVAND; PARKER, 2004; JUEL et al., 2004; MENZIES et al., 20 I0). Dessa forma, altos valores de lactato sanguíneo pós-exercício indicariam não apenas uma alta taxa de mobilização das vias anaeróbias, mas também uma alta capacidade de remoção muscular e co-transporte de íons $\mathrm{H}^{+}$, os quais podem estar relacionados com a redução da capacidade contrátil (JUEL et al., 2004; KEMP et al., 2006).

No presente estudo, a cinética de remoção de lactato apontou um aumento significativo dos valores nos momentos Pós 0', 5' e 20' em relação à situação Pré (Figura I). As altas concentrações de lactato após os esforços específicos $(9,8 \mathrm{mmol} /$, 9,4 mmol// e 6,6 mmol/) ilustram uma grande participação da via glicolítica durante a prática competitiva da modalidade, como anteriormente sinalizado por outros autores (ZAMPARO et al., 2006; NIBALLI et al., 201 I).

Uma interpretação mais qualitativa foi realizada no presente estudo aplicando o tamanho do efeito de Cohen. Os resultados indicaram que entre o momento pós 20' e pós 5' houve uma redução de 9,4 para 6,6 mmol, classificada pela escala adaptada de Hopkins com efeito moderado $(-0,63)$, demonstrando que 20 minutos após o término da prova de canoagem slalom, uma considerável quantidade de lactato já tinha sido removida da corrente sanguínea. Considerando o intervalo de 90 a 120 minutos entre a primeira e a segunda descida do percurso, pode-se inferir que esse período de intervalo seria suficientemente adequado para possibilitar que os atletas estivessem em condição metabólica favorável para um bom desempenho na segunda tentativa. Investigações com judocas, avaliando a cinética de lactato após uma luta, observaram aumento significativo nos valores de lactato logo após a luta, indicando similaridade nos valores com o presente estudo (FRANCHINI et al., 2001 ; FRANCHINI et al., 2004).

Em investigação conduzida com I I canoístas do sexo masculino, finalistas do Campeonato Mundial, incluindo dois medalhistas olímpicos, realizou-se teste incremental em kayak ergômetro e os resultados apontaram valores de pico de lactato de 13,0 \pm 2,8 mmol/ (GARCÍA-PALLARÉS et al., 2009). Resultados similares nos valores de lactato pico de $13.5 \pm 2.2 \mathrm{mmol} / \mathrm{foram}$ encontrados em teste em remo ergômetro de 1000 metros com grupo experiente de I I mulheres atletas de canoagem (idade $32 \pm 9$ anos, estatura de $169 \pm 9 \mathrm{~cm}$, massa corporal $69.7 \pm 9.0 \mathrm{~kg}$ ) (SEALEY et al., 20 l 0), e ambos os estudos indicam a natureza glicolítica dos esforços.

Em outro estudo com canoagem velocidade na modalidade $K_{500}$ que, embora ocorra em água parada, diferente da canoagem slalom, o tempo de percurso em nível mundial é muito similar, sendo de aproximadamente 100 - 1 I 0 segundos por prova (BISHOP et al., 2002; MICHAEL et al., 20 I2). Os autores submeteram oito 
atletas de ambos os sexos de alto nível competitivo a teste em kayak ergômetro por 2 minutos. Os resultados apontaram um aumento crescente durante o teste, com valores pré teste de 1,4 \pm 1,3 mmol, para 3,3 \pm 1,3 após o aquecimento e I3,0 $\pm 3,2 \mathrm{mmol} / \mathrm{l}$ no pós teste (BISHOP et al., 2002). Os mesmos autores realizaram estudo semelhante com nove canoístas de velocidade do sexo feminino de alto nível competitivo com desempenho em kayak ergômetro por 2 minutos. Os resultados demonstraram alta correlação entre o desempenho total no teste de $\mathrm{K}_{500 \text { e }} \mathrm{VO}_{2}$ pico $(r=0.82 ; P<0.05)$ e limiar anaeróbico $(r=0.89 ; P<0.05)(\mathrm{BISHOP}$ et al., 2000b). Resultados similares foram encontrados por Franchini et al. (2009) com lutadores de judô. Os pesquisadores concluíram que os atletas melhor preparados anaerobiamente demonstravam remoção mais rápida do lactato sanguíneo, sendo o período de 15 minutos entre as lutas, suficiente para recuperação dos atletas altamente treinados (FRANCHINI et al., 2009).

O presente estudo apresenta como limitação um pequeno número de atletas, contudo investigações com alto nível competitivo, em geral, contam com número reduzido de sujeitos (MELLO et al., 2009; GARCIA-PALLARES et al., 20 I 0; MICHAEL et al., 20I0; MICHAEL et al., 20I2; MACIEJEWSKI et al., 20I2). Outro aspecto importante de se considerar é que os resultados são fruto de uma análise transversal, também muito comum em estudos descritivos (MELLO et al., 2009; MICHAEL et al., 20 I0; BUGLION et al., 20। I; MICHAEL et al., 20I2; MACIEJEWSKI et al., 20 I2). A despeito destas limitações, os resultados entre o melhor (86,4 segundos) e o pior tempo de prova ( 137 segundos), a cinética de redução do lactato indicou grande variação individual, similar ao descrito em outras investigações com este metabólito (BISHOP et al., 200 I ; MENZIES et al., 20 I 0; MACIEJEWSKI et al., 20 I 2; MICHAEL et al., 20 I2). Os resultados do melhor desempenho após a descida foram de 7.7 mmol//; seguido por 6.8 após 5'e de 3.9 mmol/ após 20'; enquanto que no atleta que obteve o pior desempenho na prova os valores pós foram de $11.2 \mathrm{mmol} /$; de 8,5 após 5' e de 6.7 mmol// após 20'. Estes resultados apontam que o atleta melhor preparado anaerobiamente demonstra magnitude menor na concentração de lactato pós prova e uma remoção mais rápida nos momentos pós chegada, corroborando com relatos prévios na literatura (JUEL et al. 2004), e demonstrando como foco o treinamento de alta intensidade para atletas dessa modalidade, tal como o sinalizado por MACIEJEWSKI et al. (20 I2), que relataram a contribuição glicolítica na ressíntese total de ATP total durante o exercício supramáximo.

\section{CONCLUSÃO}

Os principais achados do presente estudo caracterizam os atletas brasileiros da canoagem slalom e descrevem a prova competitiva com alta contribuição glicolítica. A 
partir da descrição da cinética de produção e remoção de lactato, é possível observar que os atletas com melhor desempenho tiveram maior magnitude de remoção do metabólito imediatamente após a descida e 5 minutos após, o que pode sugerir que essa capacidade de remoção está associada à condição metabólica, que predispõe a um alto desempenho nessa modalidade. Esses achados são os primeiros indicadores da modalidade, e denotam aspectos interessantes que nos remetem a sugerir futuros estudos com foco na elucidação de outros fatores fisiológicos da competição (por exemplo: consumo de oxigênio) e suas repercussões sobre a preparação dos atletas.

\section{Analysis of the Kinetics of Lactate Removal in Slalom Kayak Athletes}

ABSTRACT: The aim of this study was to verify the kinetics of lactate production and removal in slalom kayak athletes, KI category, during official competition. Eight male athletes $(22,6$ $\pm 4,3$ years) participated of the study. For the analysis of lactate, $25 \mu \mathrm{L}$ of capillary blood were collected. The kinetics of lactate removal was performed before the warm-up (Pre), just after the competitors exit from the river (Post 0'), 5 (Post 5'), and 20 (Post 20') minutes. The results demonstrated a significant increase in lactate concentrations (9.8 mmol/l, 9.4 $\mathrm{mmol} / \mathrm{l}$ and $6.6 \mathrm{mmol} / \mathrm{l})$ at $0^{\prime}, 5^{\prime}$ and $20^{\prime}$ post respectively, with values of $P<0.01$. The findings indicate that after 20 minutes the values of lactate reduced significantly $(P<0.05)$ compared to Pre exercise, suggesting that the athletes would indicate good metabolic conditions for the second turn of the race.

KEYWORDS: Blood lactate; high-intensity exercise; competition; Athletes

\section{Análisis de la cinética de la eliminación de lactato de atletas de piragüismo slalom}

RESUMEN: El objetivo de este estudio fue examinar la cinética de la producción y la remoción de lactato en atletas de slalom piragüismo, categoría KI, durante la competición oficial. En el estudio participaron ocho hombres (22,6 \pm 4,3 años), con $25 \mu \mathrm{L}$ de muestras de sangre para el análisis de lactato. La cinética de eliminación de lactato se realizó antes del calentamiento (Pre), poco después de la salida de los competidores del rio (Post 0'), 5 (Post 5') y 20 (Post 20') minutos. Los resultados mostraron un aumento significativo en la concentración lactato (9.8 mmol/l, 9.4 mmol/l e $6.6 \mathrm{mmol} / \mathrm{l}$ ) en los tiempos 0', 5' e 20' minutos respectivamente, con valores de $P<0,01$. Resultados indican que después de 20 minutos los valores de lactato se redujeron significativamente $(P<0,05)$ en comparación con el ejercicio anterior, lo que sugiere buenas condiciones metabólicas para el segundo descenso de la prueba.

PALABRAS-CLAVE: Lactato de la sangre; alta intensidad de ejercicio; competencia; atletas. 


\section{REFERÊNCIAS}

AKCA, F; MUNIROUGLU, S. Anthropometric-Somatotype and Strength Profiles and On-Water Performance in Turkish Elite Kayakers. International Journal of Applied Sports Sciences, Korea, v. 20, n. I, p. 22-34, 2008.

BOGDANIS, G.; C. Effects of physical activity and inactivity on muscle fatigue. Frontiers in Physiology, Lausanne, v.3, p. 2-15, may. 2012.

BISHOP, D. Physiological predictors of flatwater kayak performance in women. European Journal of Applied Physiology, Berlin, v.82, p. 91-97, 2000a.

BISHOP, D. Physiological predictors of at-water kayak performance in women. European Journal Applied Physiology, Berlin, v. 82, p. 91-97, 2000b.

BISHOP, D.; BONETTI, D.; DAWSON, B. The effect of three different warm-up intensities on kayak ergometer performance. Medicine and Science in Sports and Exercise, Madison, v. 33, n. 6, p. $1026-1032,2001$.

BISHOP, D.; BONETTI, D.; DAWSON, B. The influence of pacing strategy on $\mathrm{VO}_{2}$ and supramaximal kayak performance. Medicine and Science in Sports and Exercise, Madison, v. 34, n. 6, p. 1041-1047, 2002.

BISHOP, D.; CLAUDIUS, B. Effects of induced metabolic alkalosis on prolonged intermittent-sprint performance. American College of Sports Medicine, Philadelphia, v. 37, n. 5, p. 759-767, 2005.

BISHOP, D.; EDGE J.; THOMAZ, C.; MERCIER J. Effects of high-intensity training on muscle lactate transporters and postexercise recovery of muscle lactate and hydrogen ions in women. American Journal Physiology Regulation and Comp Physiology, Bethesda, v. 295, p. RI99I-R|998, 2008.

BONEN, A. et al. Short-term training increases human muscle MCTI and femoral venous lactate in relation to muscle lactate. American Journal of Physiology- Endocrinology and Metabolism, Bethesda, v. 274, n. I, p. 102-107, 1998.

BONETTI, D.; HOPKINS, W. G.; KILDING, A. E. High-intensity kayak performance after adaptation to intermittent hypoxia. International Journal of Sports Physiology and Performance, Champaign, v. I, p. 246-260, 2006.

BONEN, A. Lactate transporters (MCT proteins) in heart and skeletal muscles. Medicine and Science in Sports and Exercise, Madison, v. 32, n. 4, p. 778-89, April. 2000.

BRET, C. et al. Differences in lactate exchange and removal abilities in athletes specialized in different track running events ( 100 to 1500 m). International Journal Sports Medicine, Stuttgart, v. 24, n. 2, p. 108-13, February. 2003. 
CABRERA, ME.; SAIDEL, G. M.; KALHAN, S. C. Lactate metabolism During Exercise: analysis by an integrative systems model. American Journal Physiology Regulation and Comp Physiology, Bethesda, v. 277, p. 1522-1536, 1999.

CAIRNS, S. P. Lactic Acid and Exercise: Culprit or Friend? Sports Medicine, Auckland, v. 36, n. 4, p. 279-29I, 2006.

CERRETELLI, P., SAMAJA, M.: Acid-base balance at exercise in normoxia and chronic hypoxia. Revisiting the lactate paradox. European Journal of Applied Physiology, Berlin, v. 90, p. 43 I-448, 2003.

CBCA - Confederação Brasileira de Canoagem. Site Oficial da CBCa. Disponível em < http:// www.cbca.org.br. Acesso em 19 setembro de 2012.

COHEN, J. Statistical Power Analysis for the Behavioral Sciences. Second Edition ed. Hillsdale: Lawrence Erlbaum Associates 1988.

FELL, J. W. et al. Evaluation of the Accusport a lactate analyser. International Journal Sports Medicine, Stuttgart,v. 19, n. 3, p. 199-204, 1998.

FRANCHINI, E. et al. Efeito do tipo de recuperação após uma luta de judô sobre o desempenho anaeróbio. Corpo Consciência, Rio Preto, v. 7, p. 23-39, Jan/Jun. 2001.

FRANCHINI, E. et al. Nível Competitivo, tipo de recuperação e remoção do lactato após uma luta de judô. Revista Brasileira de Cineantropometria e Desempenho Humano, Santa Catarina, v.6, n. I, p. 7-16, maio. 2004.

FRANCHINI, E. et al. Effects of recovery type after a judo match on blood lactate and performance in specific and non-specific judo tasks. European Journal Applied Physiology, Berlin, n. 107, p. 377-383, july. 2009.

GARCÍA -PALLARÉS, J. et al. Endurance and neuromuscular changes in world-class level kayakers during a periodized training cycle. European Journal of Applied Physiology, Berlin, v. 106, p. 629-638, april. 2009.

GARCÍA -PALLARÉS, J. et al. Performance changes in world-class kayakers following two different training periodization models. European Journal of Applied Physiology, Berlin, v. I I0, v. I, p. 99-107, september. 2010a.

GARCÍA -PALLARÉS, J. et al. Physiological effects of tapering and detraining in world-class kayakers. Medicine in Science in Sports and Exercise, Madison, v. 42, n.6, p. 1209-1214, may. $2010 b$

GARCÍA -PALLARÉS, J., IZIQUIERDO, M. Strategies to optimize concurrent training of strength and aerobic fitness for rowing and canoing. Sports Medicine, Auckland, v.4I, n. 4, p.329-343, april. 2011 . 
GLADDEN, L. B. Muscle as a consumer of lactate. Medicine and Science in Sports and Exercise, Madison, v. 32, n. 4, p. 764-7I, april. 2000.

GLADDEN, L. B. Is there an intracellular lactate shuttle in skeletal muscle? Journal Physiology, Sydney, v. 582, n. 3, p. 899, august. 2007.

GOMES, B. B. et al. Gross efficiency and energy expenditure in kayak ergometer exercise. International Journal of Sports Medicine, Stuttgart, v.33., n. 8., p. 654-660, august. 20I2.

HARRISON GG. et al. Skinfold thicknesses and measurement technique. In: Lohman TG, Roche AF, Martorell R, editors. Anthropometric standardization reference manual. Champaign: Human Kinetics Books, 1988; 55-70.

HENSEL, P.; PERRONI, M. G.; LEAL JÚNIOR, E. C. P. Lesões musculoesqueléticas na temporada de 2006 em atletas da seleção brasileira feminina principal de canoagem velocidade. Acta Ortopedica Brasileira, São Paulo, v. 16, n. 4, p. 233-237, dezembro. 2008.

HOPKINS, W.G. et al. Progressive statistics for studies in sports medicine and exercise science. Medicine and Science in Sports and Exercise, Madison, v. 4I, n. I, p. 3-13, 2009.

HUNTER, A. Canoe slalom boat trajectory while negotiating an upstream gate. Sports Biomechanics, Edinburgh, v. 8, n. 2, p. 105-113, june. 2009.

INTERDONATO, R.S.; LUETKEMEIER, M. J. Reliability and validity of venous blood lactate measurement with Accusport portable lactate analyzer. Medicine and Science in Sports and Exercise, Madison, v.3I, n. 5, p. s353, 1999.

JACKSON, A. S.; POLLOCK, M. L. Generalized equations for predicting body density of man. Brazilian Journal of Nutrition, São Paulo, v.40, n.3, p. 497-504, november. 1978.

JUEL, C. et al. Effect of high-intensity intermittent training on lactate and $\mathrm{H}^{+}$release from human skeletal muscle. American Journal of Physiology and Endocrinology and Metabolism, Bethesda, v. 286, p. E245-E25I, 2004.

KEARNEY, J.T.; MCKENZIE, D. C. Physiology of canoe sport. In: GARRETT, W. E.; KIRKENDALL, D. T. (eds). Exercise and Sport Science. Philadelphia: Lippincott, Williams and Williams, 2000, p. 745-757.

KEMP, G. et al. Explaining pH change in exercising muscle: lactic acid, proton consumption, and buffering vs. strong ion difference. American Journal Physiology Regulation and Comp Physiology, Bethesda, v. 29I, p. R235-237, 2006.

LEMOS, L. F. C.; TEIXEIRA, C. S.; MOTA, C. B. Lombalgia e o equilíbrio corporal de atletas da seleção brasileira feminina de canoagem velocidade. Revista Brasileira Cineantropometria e Desempenho Humano, Santa Catarina, v. 12, n. 6, p. 457-463, nov/dez. 2010.

MACIEJEWSKI, H. et al. Lactate accumulation in response to supramaximal exercise in rowers. Scandinavian Journal of Medicine \& Science in Sports, Copenhagen, janeiro. 2012. 
MICHAEL, J. S., SMITH, R., ROONEY, K. B. Determinants of kayak paddling performance. Sports Biomechanics, Edinburgh, v 8, n. 2, p: 167-179, june. 2009.

MICHAEL, J. S.; ROONEY, S. K. Physiological Responses to Kayaking with a Swivel Seat. International Journal of Sports Medicine, Stuttgart, v.3 I, p. 555 - 560, march. 2010.

MICHAEL, J. S., ROONEY, K. B.; SMITH, R. The dynamics of elite paddling on a kayak simulator. Journal of Sports Sciences, London, v.30, n.7, p. 66I-668, april. 2012.

MESSONNIER, L. et al. Effects of training on lactate kinetics parameters and their influence on short high-intensity exercise performance. International Journal of Sports Medicine, Stuttgart, v. 27, n. I, p. 60-6, january. 2006.

MELLO, F. C. et al. Energy systems contributions in 2,000 m race simulation: a comparison among rowing ergometers and water. European Journal of Applied Physiology, Berlin, v. 107 , p. 615-619, august. 2009.

MENZIES, P. et al. Blood lactate clearance during active recovery after an intense running bout depends on the intensity of the active recovery. Journal of Sports Sciences, London, v. 28, n. 9, p. 975-982, july. 2010.

MOREIRA, A. et al. Esforço percebido, estresse e inflamação do trato respiratório superior em atletas de elite de canoagem. Revista Brasileira Educação Física e Esporte, São Paulo, v. 23, n.4, p.355-63, out/dez. 2009.

NAKAMURA, F. Y. et al. Perfil fisiológico de canoístas do sexo feminino de alto nível competitivo. Revista Portuguesa Ciência do Desporto, Porto, v. 6, n. 3, p: 329-335, out. 2006.

NAKAMURA, F. Y. et al. Relação entre indicadores fisiológicos obtidos em teste ergoespirométrico em cicloergômetro de membros superiores e desempenho na canoagem. Revista Brasileira de Medicina do Esporte, Rio de Janeiro, v. I3, n. 5, p. 283- 286, set/out. 2007.

NIBALI, M.; HOPKINS, W. G.; DRINKWATER, E. Variability and predictability of elite competitive slalom canoe-kayak performance. European Journal of Sport Science, v. I I, n.2, p. I25-130, march. 201।.

ROBERGS, R. A. Exercise-induced metabolic acidosis: where do the protons come from? Sport Sciences, London, v. 5, n. 2, 2001.

ROBERGS, R. A.; GHIASVAND, F.; PARKER, D. Biochemistry of exercise-induced metabolic acidosis. American Journal Physiology and Regulation Integral Comp Physiology, Bethesda, v. 287, n. 3, p. R502-16, september. 2004.

SEALEY, R. M. et al. Identification and reliability of pacing strategies in outrigger canoeing ergometry. Journal of Science and Medicine in Sport, Bursa, v. 13, p. 24I-246, 2010.

SIRI, WE. (|96I). Body composition from fluid spaces and density: analysis of methods. In: BROZECK, ..; HENSCHEL, A. (Eds.). Techniques for measuring body composition. Washington, DC: National Academy of Science. p. 233-244 
THOMAS, C. et al. Monocarboxylate transporters, blood lactate removal after supramaximal exercise, and fatigue indexes in humans. Journal of Applied Physiology, Bethesda, n. 98, p. 804-809, 2005.

ZAMPARO, P. et al. Bioenergetics of a Slalom kayak (KI) Competition. International Journal of Sports Medicine, Stuttgart, v. 27, n. 7, p 546-552, 2006.

Recebido em: 24 ago. 2011 Aprovado em: 16 nov. 2012

Endereço para correspondência: Carla Cristiane da Silva Centro de Ciências da Saúde Departamento de Educação Física Alameda Padre Magno, 84I Jacarezinho/PR (43)35250498

Cx. Postal 26I - CEP 86400-000 